\title{
Image Processing to Estimate the Heat Sources Related to Phase Transformations during Tensile Tests of NiTi Tubes
}

\author{
P. Schlosser*t, H. Louche ${ }^{\dagger}$, D. Favier* and L. Orgéas* \\ *Laboratoire Sols-Solides-Structures, CNRS, Universités de Grenoble (INPG - UJF), BP 53, F-38 04I Grenoble Cedex 9, France \\ †Laboratoire SYMME, Polytech'Savoie, Université de Savoie, BP 80439, F-74944 Annecy Le Vieux Cedex, France
}

\begin{abstract}
Temperature and strain fields have been observed during superelastic tensile tests on $\mathrm{NiTi}$ tubes. They show strong localisations that take the shape of helical bands with characteristics that depend on global tensile strain and strain rate. To obtain quantitative energy information, allowing a better recognition of the deformation mechanisms involved inside and outside the helical bands, an estimation of the local heat sources based on image processing of the temperature fields is proposed in the present paper. This processing method is first calibrated and validated on numerically simulated temperature fields calculated using theoretical heat sources close to those associated with martensitic phase transformation. It is then applied to experimentally observed temperature fields during a typical superelastic tensile test of an $\mathrm{NiTi}$ tube.
\end{abstract}

KEY WORDS: heat sources estimation, localisation, NiTi shape memory alloy, superelastic tensile tests, thermography

\begin{tabular}{|c|c|c|c|}
\hline \multicolumn{4}{|l|}{ NOTATIC } \\
\hline$L_{0}$ & $\begin{array}{l}\text { Gauge length of the tube deformed in } \\
\text { tension }(\mathrm{m})\end{array}$ & $\frac{\partial f}{\partial x}=f_{, x}$ & $\begin{array}{l}\text { Partial derivative of the scalar function } f \\
\text { with respect to the scalar } x\end{array}$ \\
\hline$R, e$ & Mean radius and thickness of the tube (m) & $T_{0}=T_{a}$ & Homogeneous initial tube temperature $(\mathrm{K})$ \\
\hline$T_{a}$ & Ambient temperature $(\mathrm{K})$ & $T(\mathbf{x}, t)$ & Temperature spatial field (K) \\
\hline \multirow[t]{2}{*}{$t_{0}, t$} & \multirow{2}{*}{$\begin{array}{l}\text { Reference and current times, respectively } \\
\text { (s) }\end{array}$} & $\theta=T-T_{\mathrm{o}}$ & Temperature variation spatial field (K) \\
\hline & & \multirow[t]{3}{*}{$<T>,<\theta>$} & \multirow{3}{*}{$\begin{array}{l}\text { Average temperature and temperature } \\
\text { variations in the thickness of the tube at } \\
\text { given angle } \alpha \text { and height } \mathrm{z}(\mathrm{K})\end{array}$} \\
\hline$F(t)$ & Axial force $(\mathrm{N})$ & & \\
\hline$U(t)$ & Crosshead displacement (m) & & \\
\hline$\dot{\varepsilon}=\dot{U} / L_{0}$ & Constant nominal axial strain rate $\left(\mathrm{s}^{-1}\right)$ & $\delta T=T-<T>$ & Radial temperature fluctuation $(\mathrm{K})$ \\
\hline$X$ & Particle (or material point) & $s_{i}(\mathbf{x}, t)$ & \\
\hline $\mathrm{M}_{0}$ and $\mathrm{M}$ & $\begin{array}{l}\text { Spatial points occupied by the particle } X \text { at } \\
t_{0} \text { and } t \text {, respectively }\end{array}$ & $s_{e}(\mathbf{x}, t)$ & $\begin{array}{l}\text { Internal heat source per unit volume of } \\
\text { internal and external origin, respectively } \\
\left(\mathrm{W} . \mathrm{m}^{-3}\right)\end{array}$ \\
\hline $\begin{array}{l}\mathrm{OM}_{0}=\mathrm{X} \\
\mathrm{OM}=\mathrm{x}\end{array}$ & \multirow[t]{2}{*}{$\begin{array}{l}\text { Reference and current positions of } X \\
\text { relative to an origin } \mathrm{O} \text {, respectively }\end{array}$} & $<\mathrm{S}_{i}>,<\mathrm{s}_{e}>$ & $\begin{array}{l}\text { Average value of } s_{i}(\mathbf{x}, t) \text { and } s_{e}(\mathbf{x}, t) \text { in the } \\
\text { thickness of the tube }\left(\mathrm{W} \cdot \mathrm{m}^{-3}\right)\end{array}$ \\
\hline \multirow{3}{*}{$\begin{array}{l}f(\mathbf{X}, t) \\
\text { and } \\
f(\mathbf{x}, t)\end{array}$} & & $\begin{array}{l}\mathbf{u}(\ldots, t)= \\
\mathbf{M}_{\mathbf{0}} \mathbf{M}\end{array}$ & Displacement field at time $t(\mathrm{~m})$ \\
\hline & \multirow{2}{*}{$\begin{array}{l}\text { Material and spatial descriptions for any } \\
\text { scalar, vectorial or tensorial field }\end{array}$} & $\mathbf{v}(\ldots, t)=\frac{D \mathbf{u}}{D t}$ & Velocity field at time $t\left(\mathrm{~m} . \mathrm{s}^{-1}\right)$ \\
\hline & & $\rho, \mathrm{k}$ & Mass density $\left(\mathrm{kg} \cdot \mathrm{m}^{-3}\right)$ and thermal \\
\hline$\frac{D f}{D t}=\dot{f}$ & $\begin{array}{l}\text { Material time derivative of a scalar, vecto- } \\
\text { rial or tensorial function }\end{array}$ & $C$ & $\begin{array}{l}\text { conductivity }\left(\mathrm{W} \cdot \mathrm{m}^{-1} \cdot \mathrm{K}^{-1}\right) \text {, respective } \\
\text { Specific heat capacity }\left(\mathrm{J} \cdot \mathrm{kg}^{-1} \cdot \mathrm{K}^{-1}\right)\end{array}$ \\
\hline
\end{tabular}

\section{Introduction}

Superelastic shape memory alloys (SMA) are increasingly being used in a large number of applications in the fields of aeronautical, biomedical and structural engineering. Superelasticity refers

to the ability of a material in a given temperature range to accommodate relatively large inelastic strains during loading and then recover all of the strain upon unloading, via a hysteresis loop, as shown in Figure 4 . The underlying mechanism of superelasticity is a diffusionless first-order reversible 
solid-solid phase change. The individual grains in polycrystalline SMAs can abruptly change their lattice structure in the presence of suitable thermomechanical loading. The transformation can be induced by changes in temperature or by changes in stress because of the strong thermomechanical coupling in the material behaviour. For stressinduced transformations, this deformation mechanism leads to a superelastic behaviour highly dependent on temperature.

As a result of their outstanding superelastic behaviour at human body temperature and their biocompatibility, polycrystalline Ti-50.8 at.\% Ni SMA are increasingly being used for biomedical applications (human implants and surgery instruments). Components and devices that utilise the peculiar properties of these NiTi SMA are increasingly being designed using numerical simulation and finite element software, in which three-dimensional constitutive equations are implemented to model the thermomechanical behaviour of the material. These constitutive equations are mostly based on the nominal stress-strain curves of specimens deformed in tension, as shown in Figure 4. It is now well established that superelastic tension tests on polycrystalline NiTi SMA plates, wires or tubes often exhibit localised Lüders-like deformation modes [1]. The localisation has been studied using qualitative optical observations [1-3], multiple extensometers [4] and full-field temperature measurements [5]. It is thus not possible to deduce directly constitutive equations for NiTi SMAs from tensile curves by assuming, as is usual, a homogeneous deformation. Therefore, a deeper understanding of these localisation phenomena is required.

To enable a better understanding as mentioned above, this paper describes the developments of a numerical software that allows thermal analysis of the superelastic behaviour. This software gives an estimation of the local heat sources based on image processing of the temperature fields. The material properties, test procedures and results are described in the succeeding section. The section 'Heat Source Estimation' develops the theory and describes the assumptions used in the data processing. The validation is based on numerically simulated temperature fields that are calculated using theoretical heat sources close to those obtained experimentally during the martensitic phase transformation. Finally, the section 'Example of Results' gives results obtained by applying the processing to experimentally observed temperature fields during a typical superelastic tensile test of an NiTi thin tube.

\section{Material, Equipments, Experimental Procedure and Results}

The specimens used in the experiments were commercially available polycrystalline Ti-50.8 at.\% Ni thin tubes of inner and outer diameters of 10 and $11 \mathrm{~mm}$ respectively. These tubes were manufactured by Minitubes SA (Grenoble, France) using multiple mechanical and thermal treatments. The final cold drawing and the subsequent annealing treatment $(\approx 773 \mathrm{~K}$ for $15 \mathrm{~min}$ ) give the tubes their final superelastic properties at human body temperature and a very small grain size (few microns).

Differential Scanning Calorimetry (DSC) measurements shown in Figure 1 exhibit a two-stage transformation: austenite (A) $\rightarrow$ R-phase $(\mathrm{R}) \rightarrow$ martensite (M) and $\mathrm{M} \rightarrow \mathrm{R} \rightarrow \mathrm{A}$ upon free stress cooling and heating, respectively. On cooling, the transformation $\mathrm{A} \rightarrow \mathrm{R}$ begins at approximately $T(\mathrm{~A}-\mathrm{R})_{\mathrm{s}}=301 \mathrm{~K}$ with a peak at $289.3 \mathrm{~K}$. It is completed at $T(\mathrm{~A}-\mathrm{R})_{\mathrm{f}}=278 \mathrm{~K}$. With further cooling, the transformation $\mathrm{R} \rightarrow \mathrm{M}$ begins at $226 \mathrm{~K}$ and is not complete at $183 \mathrm{~K}$. Upon heating, both transformations are present, but the temperature ranges for the reverse transformation of the R-phase and M-phase overlap. The transformation $\mathrm{M} \rightarrow \mathrm{R}$ starts at $T(\mathrm{R}-\mathrm{M})_{\mathrm{s}}=226 \mathrm{~K}$, the peak occurs at $280 \mathrm{~K}$, and the reverse transformations $\mathrm{R} \rightarrow \mathrm{A}$ are completed at $301 \mathrm{~K}$ with a peak at 295.7 K.

A specially designed gripping system (Figure 2b) was created using a pair of standardised collets and collet chuck (2) with a pin inside to avoid crushing the tube (1) [6]. Both collet and chuck are linked to an universal mechanical tensile testing machine (Instron 5569, 50 kN; Instron, High Wycombe, UK) via two Cardan joints (3) to avoid any bending of the tensile specimen. During the tests, the usual

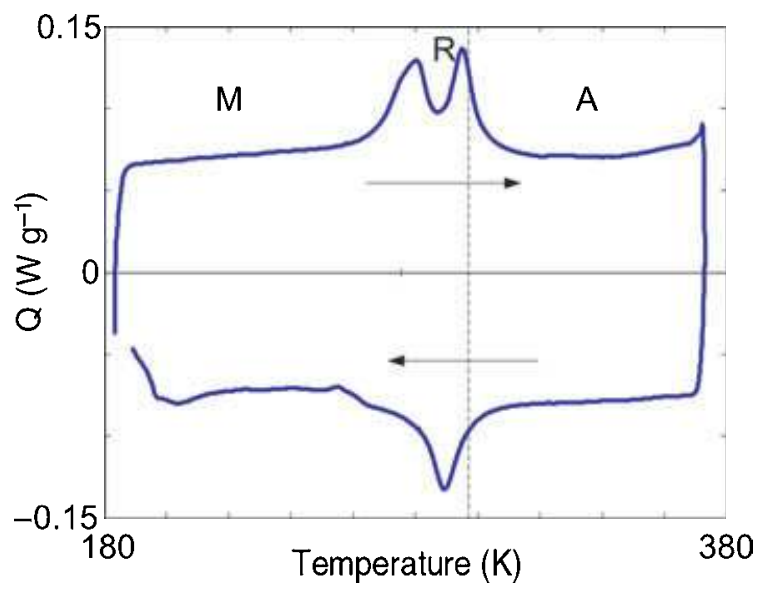

Figure I: DSC measurements of the material. The dashed line indicates the initial homogeneous temperature of the tube $\left(T_{0}=297 \mathrm{~K}\right)$ equal to the ambient temperature 
(A)

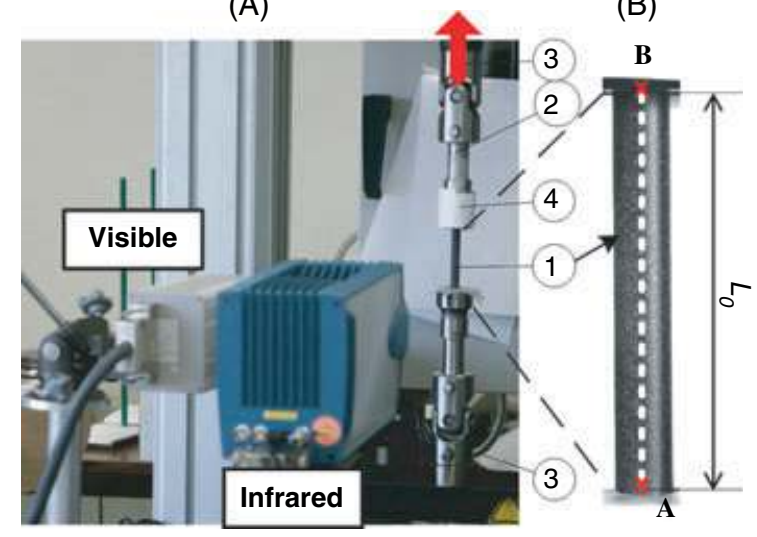

Figure 2: (A) Experimental setup with the two infrared and visible digital cameras, (B) view of the griping system with the tube (1) in the centre

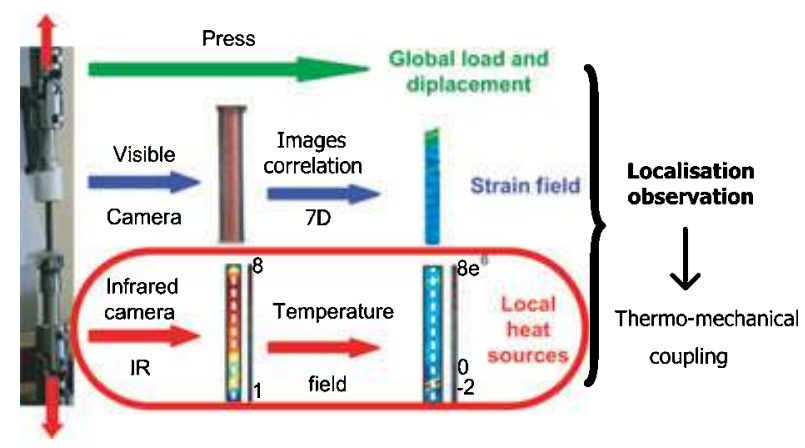

Figure 3: Measurement and data management

mechanical data (axial force $F$, crosshead displacement $U$ ) as well as the thermal and kinematic fields were acquired simultaneously (Figure 2A) with two digital cameras, one infrared and the other visible (Figure 3).

The displacement field of any particle of the outer surface of the tube was obtained using a digital visible camera (Hamamatsu, $1280 \times 1024$ pixels, $9 \mathrm{~Hz}$, ambient lighting; Hamamatsu Photonics Ltd, Welwyn Garden City, UK) and an image correlation processing software ('7D' software [7]). The temperature field was obtained by using a fast multidetector digital infrared camera (CEDIP Jade III MW, $145 \mathrm{~Hz}$; CEDIP Infrared Systems, Croissy Beaubourg, France) which has a resolution of $320 \times 240$ pixels. The spatial resolution (pixel size), that depends on the adjustment of the focal distance, was estimated to be close to $0.4 \mathrm{~mm}$ for the tests carried out with the infrared camera. Prior to the tests, the surface of the tube was coated with a highly emissive paint in order to obtain the blackbody properties compatible with the calibration law of the camera, with an accuracy on temperature variations of $<0.1 \mathrm{~K}$.

The typical measured macroscopic nominal stressnominal strain curve of the tube during loading and

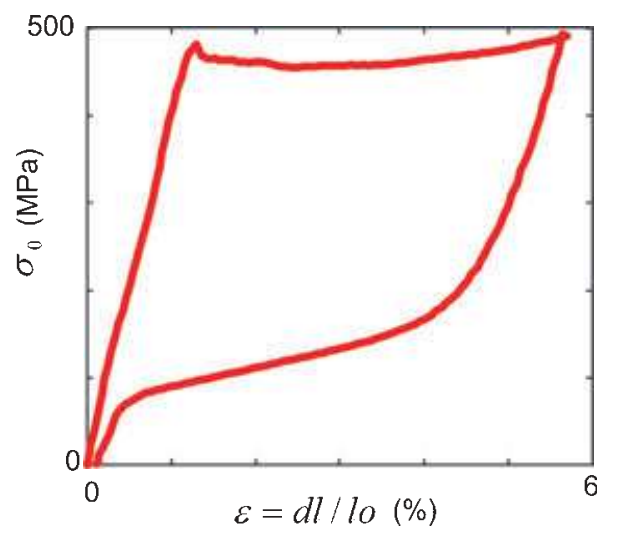

Figure 4: Nominal stress-strain curve during tensile loading and unloading

unloading is shown in Figure 4. This tensile test was carried out at room temperature at a constant crosshead velocity of $\dot{U}=1 \mathrm{~mm} \mathrm{m^{-1 }}$. A tube of $L_{0}=$ $71 \mathrm{~mm}$ in gauge length was used (Figure 2B). The nominal axial strain rate is defined as $\dot{\varepsilon}=\dot{U} / L_{0}=$ $2.35 \times 10^{-4} \mathrm{~s}^{-1}$. Before being clamped, the tube was heated up to $373 \mathrm{~K}$ and cooled down to the ambient temperature $\left(T_{\mathrm{a}}=297 \mathrm{~K}\right.$; see the dashed line in Figure 1), just above $T(\mathrm{~A}-\mathrm{R})_{\mathrm{s}}$. The initial tube temperature $T_{0}$ is homogeneous and equal to ambient temperature $T_{\mathrm{a}}$ so that the material is in the austenitic state with a small amount of initial R-phase and exhibits superelastic behaviour under stress.

The nominal stress is obtained by dividing the measured force by the reference cross-section of the tube. The nominal strain is obtained by dividing the difference of displacements of the two points $\mathrm{A}$ and $\mathrm{B}$ (see Figure $2 \mathrm{~B}$ ) by the gauge length $L_{0}$; the displacements were determined through image correlation processing. The 'plateau' observed during loading (and unloading) (Figure 4) is associated with localisation phenomena in which the inclined helical bands are formed during the exothermic (and endothermic) phase transformations. This results in a highly heterogeneous temperature field (Figure 3). The estimation of the associated heat sources would provide precious quantitative information and data for future theoretical development concerning modelling of the thermomechanical behaviour of NiTi SMA.

\section{Heat Source Estimation}

It is very difficult to analyse quantitatively the observed heterogeneous deformation mechanisms from temperature maps such as those shown in Figure 3. Instead, the determination of local heat source fields is much more appropriate, as deformation mechanisms in NiTi are closely linked with stress-induced 
phase transformations. This section briefly describes the method adopted to estimate these heat sources. A first set of assumptions are stated in order to write the heat balance equation describing heat transfer in the tubes (section 'Heat Balance Equation'). Accounting for the reduced wall thickness of the tubes, the heat balance equation is simplified, leading to a $2 \mathrm{D}$ formulation of the thermal problem (section 'Approximation of Heat Transfer in the Tubes: 2D Formulation). Such a formulation involves a characteristic time, indicating the natural radial heat loss which is determined experimentally (section Estimation of the Characteristic Time $\left.\tau_{t h}\right)$. The image-processing method that uses the 2D thermal formulation is described (section 'Image Processing to Calculate the Heat Source Fields from the Measured Temperature Fields'), calibrated and validated using finite element simulation (section 'Validation of the Method and Calibration of the Filters').

\section{Heat balance equation}

At each instant, $t$, the heat balance equation governing heat transfers inside the deformed tubes is deduced from the first and second laws of thermodynamics applied to continuum media, which can be written either in material or spatial local forms. The spatial local form of the first law combined with the law of balance of kinetic energy leads to the energy equation. This equation introduces the specific internal energy $e$, the mass density $\rho$, the stress power per unit volume $p_{\text {int }}$, the heat flow vector $\mathbf{q}$ per unit area, and distributed heat sources of external origins of value $s_{\mathrm{e}}$ per unit volume (radiation here):

$\rho \dot{e}=-p_{\text {int }}+s_{\mathrm{e}}-\operatorname{div} \mathbf{q}$.

It is assumed that heat conduction is governed by the standard Fourier's law, with an isotropic, uniform and constant thermal conductivity $k$. Introducing the specific heat $C$, assumed also as uniform and constant, the previous equation can be rewritten as a local heat balance equation which reads in its local form:

$\rho C \dot{T}-k$ lap $T=s_{\mathrm{i}}+s_{\mathrm{e}}$,

where 'lap' stands for the 3D Laplacian operator and $s_{\mathrm{i}}$ includes all rates of heat generation per unit volume of internal origin such as intrinsic dissipation [8, 9], thermoelastic couplings or latent heat because of solid-solid phase transformation [10, 11]. Using a cylindrical coordinate system $(r, \alpha, z)$ associated with the local reference frame $\left(M, \mathbf{e}_{r}, \mathbf{e}_{\alpha}, \mathbf{e}_{z}\right)$, the Laplacian operator is expressed as:

lap $T=\frac{1}{r} T_{, r}+T_{, r r}+\frac{1}{r^{2}} T_{, \alpha \alpha}+T_{, z z}$.

Notice that if the temperature field is expressed as a function of the spatial position $M$ of the particle $X$, i.e. $T(M, t)$, the material time derivative of the temperature is then given by:

$\dot{T}=T_{, t}+\mathbf{v} \cdot \operatorname{grad} T$,

with $\mathbf{v}$ being the velocity of the considered particle at the spatial position $M$. The second term on the righthand side of Equation (4) implies that it is necessary to know simultaneously the thermal and kinematic fields. In the following, this term will be assumed as negligible: such a strong assumption should be valid only for low velocities and/or for small temperature gradients. With all the above assumptions, the local heat conduction equation reads:

$\rho C T_{, t}-k$ lap $T=s_{\mathrm{i}}+s_{\mathrm{e}}$.

Approximation of heat transfer in the tubes: 2D formulation

The heat balance equation (5) clearly shows that heat sources [right-hand side of Equation (5)] can be theoretically deduced if the temperature field and its time and spatial (Laplacian) derivatives are known. Unfortunately, the infrared camera allows measurements of temperatures only at the outer surface of the tubes considered, so that further assumptions are required to circumvent this problem.

The following set of hypotheses are stated, as previously achieved in the case of thin flat samples $[8,9]$ :

- The thickness of the tube $e$ is small compared with its mean radius $R$ :

$e \ll R$.

- The heat supply $s_{\mathrm{e}}$ of external origin is considered as time-independent.

- The initial temperature of the tube $T_{0}$ is assumed to be homogeneous and equal to the ambient air temperature $T_{\mathrm{a}}$.

- The air temperature $T_{\mathrm{a}}$ is assumed to be constant during the whole tensile test.

- The temperature field $T$ in the tube is expressed as: 
$T(r, \alpha, z, t)=\langle T(\alpha, z, t)\rangle+\delta T(r, \alpha, z, t)$

where

$\langle\varsigma\rangle=\frac{1}{e} \int_{R-e / 2}^{R-e / 2} \varsigma \mathrm{d} r$

represents the average value of the scalar quantity $\varsigma$ in the thickness of the tube for given angle $\alpha$ and height $z$. It is supposed that:

$\delta T \ll\langle T\rangle$.

- At any given time $t$, angular position $\alpha$ and height $z$, the temperatures at the internal and external surface of the tube are assumed to be equal:

$T(R-e / 2, \alpha, z, t)=T(R+e / 2, \alpha, z, t)$.

- Introducing the notation $\langle\theta\rangle=\langle T\rangle-T_{\mathrm{a}}$, it is also assumed that

$\langle\theta\rangle \ll T_{\mathrm{a}}$

The lower the ratio $e / R$, the better the assumptions $(9,10)$. Moreover, Equation (11) implies that the heat release or absorption induced by heat sources must be small enough in order to limit the increase or decrease of $\langle T\rangle$ in the tubes.

Thereby, the heat balance equation (5) is then integrated over the thickness of the tubes and reads:

$\rho C\langle T\rangle_{, t}-k\langle$ lap $T\rangle=\left\langle s_{\mathrm{i}}\right\rangle+\left\langle s_{\mathrm{e}}\right\rangle$.

Accounting for Equation (6), the term $k\langle$ lap $T\rangle$ can be approximated by:

$k\langle\operatorname{lap} T\rangle \approx \frac{k}{e R}[T]_{R-e / 2}^{R+e / 2}+\frac{k}{e}\left[T_{, r}\right]_{R-e / 2}^{R+e / 2}+\frac{k}{R^{2}}\langle T\rangle_{, \alpha \alpha}+k\langle T\rangle_{, z z}$.

From Equation (10), the first term of the right-hand side of Equation (13) is neglected. The second term of the right-hand side of Equation (13) can be estimated from heat exchanges between the considered tube and ambient air:

$$
\begin{aligned}
\frac{k}{e}\left[T_{, r}\right]_{R-e / 2}^{R+e / 2}= & -\frac{1}{e}\left(\mathbf{e}_{\mathbf{r}} \cdot \mathbf{q}_{\mathrm{cond}}(R+e / 2, \alpha, z, t)\right. \\
& \left.-\mathbf{e}_{r} \cdot \mathbf{q}_{\mathrm{cond}}(R-e / 2, \alpha, z, t)\right),
\end{aligned}
$$

where $\mathbf{q}_{\text {cond }}(R+e / 2, \alpha, z, t)$ and $\mathbf{q}_{\text {cond }}(R-e / 2, \alpha, z, t)$ are the conductive heat flow through the outside and inside surface of the tube respectively. These heat transfers are assumed to be induced by natural convection $\left(\mathbf{q}_{\text {conv }}\right)$ and radiation $\left(\mathbf{q}_{\text {rad }}\right)$. The heat flow induced by natural convection, $\mathbf{q}_{\text {conv }}$, at the external or internal surface of the tube of respective normal $\mathbf{e}_{r}$ and $-\mathbf{e}_{r}$ can be expressed as:

$\pm \mathbf{e}_{\mathbf{r}} \cdot \mathbf{q}_{\mathrm{conv}}(R \pm e / 2, \alpha, z, t)=h\left(T(R \pm e / 2, \alpha, z, t)-T_{\mathrm{a}}\right)$,

where $h$ is a heat transfer coefficient. The heat transfer induced by radiation, $\mathbf{q}_{\mathrm{rad}}$, is expressed with the Stephan-Boltzmann law. Using Equations (9) and (11), it can be written:

$$
\begin{aligned}
\pm \mathbf{e}_{\mathbf{r}} \cdot \mathbf{q}_{\mathrm{rad}}(R \pm e / 2, \alpha, z, t) & =\sigma_{e} \varepsilon_{m} T^{4}(R \pm e / 2, \alpha, z, t) \\
& \approx \sigma_{e} \varepsilon_{m}\left(T_{0}^{4}+4 T_{0}^{3}\langle\theta\rangle\right),
\end{aligned}
$$

where $\sigma_{e}$ and $\varepsilon_{m}$ indicate the Stephan-Boltzmann constant and the surface emissivity respectively. Hence, Equation (13) is finally approximated by:

$k\langle\operatorname{lap} T\rangle \approx-\frac{2}{e}\left(h_{\mathrm{eq}}\langle\theta\rangle+\sigma_{e} \varepsilon_{m} T_{0}^{4}\right)+k \operatorname{lap}_{2}\langle T\rangle$,

where $h_{\mathrm{eq}}=h+4 \sigma_{e} \varepsilon_{m} T_{0}^{3}$ and where 'lap ${ }_{2}$ ' represents the 2D laplacian operator of the scalar averaged quantity $\langle\varsigma\rangle$ :

$\operatorname{lap}_{2}\langle\varsigma\rangle=\frac{1}{R^{2}}\langle\varsigma\rangle_{, \alpha \alpha}+\langle\varsigma\rangle_{, z z}$

At the equilibrium temperature, i.e. for $T=T_{0}=$ $\mathrm{T}_{\mathrm{a}}$, Equations (12) and (17) yield:

$\left\langle s_{\mathrm{e}}\right\rangle=\frac{2}{e} \sigma_{\mathrm{e}} \varepsilon_{m} T_{0}^{4}$.

This allows Equation (12) to be recast as:

$\rho C\left(\langle\theta\rangle_{, t}+\frac{\langle\theta\rangle}{\tau_{t h}}-d \operatorname{lap}_{2}\langle\theta\rangle\right)=\left\langle s_{\mathrm{i}}\right\rangle$,

where $d=k / \rho C$ is the thermal diffusivity and $\tau_{t h}=$ $\rho C / h_{\text {eq }}$ represents the characteristic time of the radial heat loss by convection and radiation. Hence, as shown by Equation (20), it is now possible to obtain an estimation of the averaged heat sources $\left\langle s_{\mathrm{i}}\right\rangle$, approximating the surface temperature given by the infrared camera to the averaged temperature $\langle T\rangle$ and providing proper estimations of $\tau_{t h}$ (see succeeding section), $\langle\theta\rangle_{t}$ and $\operatorname{lap}_{2}\langle\theta\rangle$ (see sections 'Image Processing to Calculate the Heat Source Fields from the Measured Temperature Fields' and 'Validation of the Method and Calibration of the Filters'). 


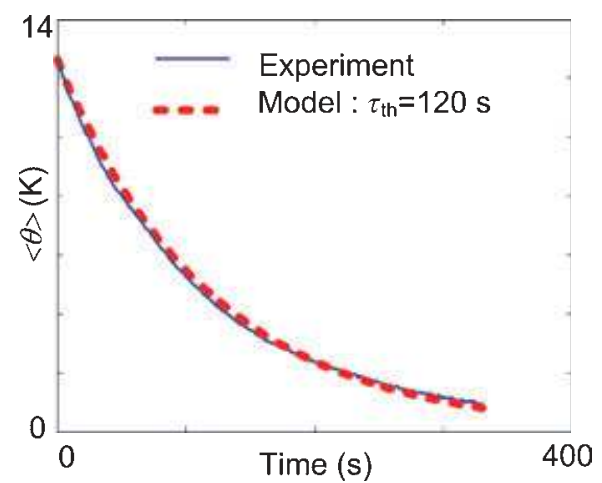

Figure 5: Experimental estimation of the characteristic time of the radial heat losses $\tau_{t h}$

\section{Estimation of the characteristic time $\tau_{\text {th }}$}

In order to estimate the characteristic time $\tau_{t h}$ of the radial heat loss by convection and radiation, an NiTi tube of length $71 \mathrm{~mm}$ was first free-stress heated up to a homogeneous temperature of $340 \mathrm{~K}$ while supported by a non-conductive rope. The ambient temperature $T_{\mathrm{a}}$ was $305 \mathrm{~K}$. From this initial state, its natural free-stress cooling from $T_{0}=335 \mathrm{~K}$ down to $323 \mathrm{~K}$ was analysed experimentally with the infrared camera. During such a cooling, the tube is fully austenitic and no phase change can occur (see Figure 1). Moreover, because of the weakness of the heat losses at the free ends of the tube, the temperature is practically homogeneous along the whole sample. Under such circumstances, Equation (20) becomes:

$\langle\theta\rangle_{, t}+\frac{\langle\theta\rangle}{\tau_{t h}}=0$,

which has the solution:

$\langle\theta\rangle=\left(T_{0}-T_{\mathrm{a}}\right) e^{\frac{-\left(t-t_{0}\right)}{\tau_{t h}}}$

The comparison of Equation (22) with experimental data for the best value of $\tau_{t h}=120 \mathrm{~s}$ is fairly good and shown in Figure 5.

Image processing to calculate the heat sources fields from the measured temperature fields

The process to estimate the heat sources fields is based on the left-hand side of Equation (20) by using the experimental temperature variation fields $\langle\theta\rangle$ [8]. These fields are obtained at the outer surface $(r=$ $R+e / 2)$ of the tube; the measures are discrete and exhibit parasitic noise. The current axial and transverse discrete coordinates of a spatial point $M$ are noted $\left(x_{i}, z_{j}\right)$ in an orthogonal Cartesian coordinate system and $\left(\alpha_{i}, Z_{j}\right)$ in a cylindrical system. The spatial

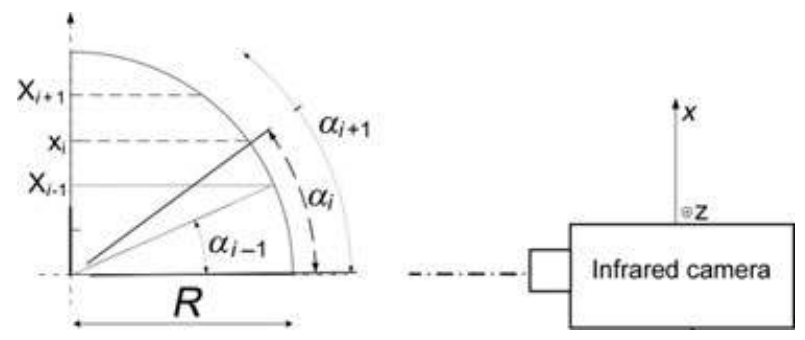

Figure 6: Link between pixel $x_{i}$ and angle $\alpha_{i}$ discretisation; $R$ is the mean radius of the tube

resolution $\Delta x=x_{i+1}-x_{i}$ and $\Delta z=z_{i+1}-z_{i}$ are the constant pixel sizes, in the two directions of the Cartesian coordinate system of the infrared camera. In our experiments this resolution was close to $\Delta x=$ $\Delta z=0.4 \mathrm{~mm}$. The spatial sampling frequency is then $1 / \Delta x=2500 \mathrm{~m}^{-1}$.

At the time $t_{k}$, the measured temperature of a spatial point can be expressed in the two coordinate systems:

$T\left(R+e / 2, x_{i}, z_{j}, t_{k}\right)=T\left(R+e / 2, \alpha_{i}, z_{j}, t_{k}\right)$,

with the relation (see Figure 6):

$x_{i}=R \sin \alpha_{i}$.

From the noisy and discrete temperature field, the heat sources are then estimated using an imageprocessing filter and discrete derivation operators, in time and space $[8,9,12]$. These derivations are estimated with a centred second-order finite difference scheme. The main difficulty is estimating the Laplacian. The image filtering, necessary to estimate operators on the noisy data, is a complex operation. It is obtained by low-pass filters applied by a fast Fourier transform. To reduce leakage effects, a regular (periodic) extension of the image is applied. The sensitivity of this method depends on the values of the cutoff frequencies. Small values are better for operator estimations but they reduce the intensity of the gradients of the unknown heat sources. An increase of these cutoff frequencies enhances the heat source detection but strongly increases the noise due to the estimation of the operators. In classical signal processing, the critical frequency is the so-called Nyquist frequency, which is half the sample frequency. In the case of a Laplacian estimation, the critical frequency needs to be smaller. Typical values of $15 \%$ of Nyquist frequencies are taken (example of various filters are detailed in the next section; Figure 14). For such cutoff frequencies and a spatial resolution of $0.4 \mathrm{~mm}$, heat sources of wavelength $<5.3 \mathrm{~mm}$ will be underestimated, i.e. the amplitude will be 
reduced. Moreover, this reduction will be associated with a spatial spreading of the source. Higher values for the cutoff frequencies would lead to significant noise in the Laplacian estimation. In order to calibrate this filtering processing, Numerically Simulated Temperature Fields (NSTF) close to the experimental data has been proposed and presented in section 'Validation of the Method and Calibration of the Filters'.

After filtering operations, the cylindrical Laplacian operator $\operatorname{lap}_{2}\langle\theta\rangle$ was calculated using a centred second-order finite differences estimation with constant discretisation steps on the vertical axis $Z$ and nonconstant steps in the $\alpha$-direction:

$$
\begin{aligned}
& \frac{\partial^{2}\langle\theta\rangle}{\partial \alpha^{2}}\left(\alpha_{i}, z_{j}, t_{k}\right) \\
& =\frac{\left(\alpha_{i}-\alpha_{i-1}\right)\left\langle\theta_{i+1}^{k}\right\rangle-\left(\alpha_{i+1}-\alpha_{i-1}\right)\left\langle\theta_{i}^{k}\right\rangle+\left(\alpha_{i+1}-\alpha_{i}\right)\left\langle\theta_{i-1}^{k}\right\rangle}{\left(\alpha_{i}-\alpha_{i-1}\right)\left(\alpha_{i+1}-\alpha_{i-1}\right)\left(\alpha_{i+1}-\alpha_{i}\right)},
\end{aligned}
$$

where $\alpha_{i}$ represent the angle of the pixel $x_{i}$, and $\left\langle\theta_{i}^{k}\right\rangle=\left\langle\theta\left(\alpha_{i}, z_{j}, t_{k}\right)\right\rangle$ the measured temperature variation at the discrete time $t_{k}$. All of these image-processing steps have been included in a software called Source2D.

\section{Validation of the method and calibration of} the filters

Overview. Numerically Simulated Temperature Fields (NSTF) have been created with the finite element software Comsol [14] in order to calibrate and filter the noise from the infrared camera and the characteristics of the phenomenon (intensity, width, velocity, etc.). Briefly, the NSTF construction is explained in Figure 7; such a method was proposed in Ref. [8]. First, thermal boundary value problems are solved on a rectangular domain representing the surface of the tube with finite element simulation involving theoretical heat sources $\left\langle s_{t h}\right\rangle$ given by a fully controlled analytical model described in the next section. Noisy temperature fields $\left\langle\theta_{N}\right\rangle$ are then created by adding artificial noise to the numerical temperature field

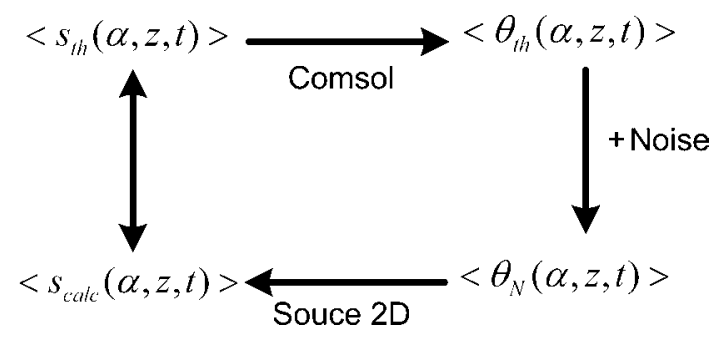

Figure 7: Organisation of the numerically simulated temperature fields (NSTF)

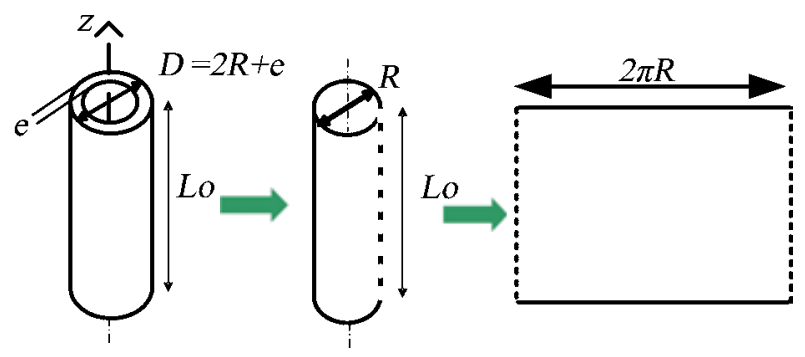

Figure 8: $2 \mathrm{D}$ thermal plate model of the tube

$\left\langle\theta_{t h}\right\rangle$. Afterwards, the noisy temperatures are used to determine the corresponding heat sources $\left\langle s_{\text {calc }}\right\rangle$ via the processing explained in the preceding subsection Finally, these estimated heat sources are compared with the theoretical sources $\left\langle s_{t h}\right\rangle$ in order to check the validity of the image processing.

The NSTF construction. Because of the small thickness of the tube, compared with the other dimensions, a $2 \mathrm{D}$ thermal plate model was built using a rectangular plane surface geometry, as shown in Figure 8:

1 Periodicity boundary conditions are imposed between the left and right sides of the domain to all the variables involved in the problem (temperature, heat flow, heat sources). Adiabatic boundary conditions were imposed to the upper and lower part of the domain.

2 In order to be as close as possible to the experiment, the theoretical heat sources $\left\langle s_{t h}\right\rangle$ were modelled using non-symmetric exponential functions (Figure 9A, Equation (26)).

$\left\langle s_{t h}(x, y)\right\rangle= \begin{cases}Q_{\max } \exp \left[-\left(\left(\frac{x-x_{p}}{a}\right)^{2}+\left(\frac{y-y_{p}}{b_{1}}\right)^{2}\right)\right] & \text { if } y>y_{P} \\ Q_{\max } \exp \left[-\left(\left(\frac{x-x_{p}}{a}\right)^{2}+\left(\frac{y-y_{p}}{b_{2}}\right)^{2}\right)\right] & \text { if } y<y_{P}\end{cases}$

This function allows us to change the width $2 a$ of the heat source band, its length $b_{1}+b_{2}$ and the velocity $V_{S}$ of its centre $P$. Choosing high values for parameters $a, b_{1}$ and $b_{2}$ will induce a diffuse phase transformation. Conversely, small values of $a, b_{1}$ and $b_{2}$, will generate phase changes in narrow bands. The centre of the heat source $P$ moves at a constant velocity $V_{S}$ along a direction $y$ oriented with an angle $\beta$ with respect to the loading direction (Figure 9B, Equation (27)):

$\left\{P(t) \mid\left\{\begin{array}{l}y_{P}(t)=V_{S}\left(t-t_{0}\right) \\ x_{p}(t)=0\end{array}\right.\right.$ 

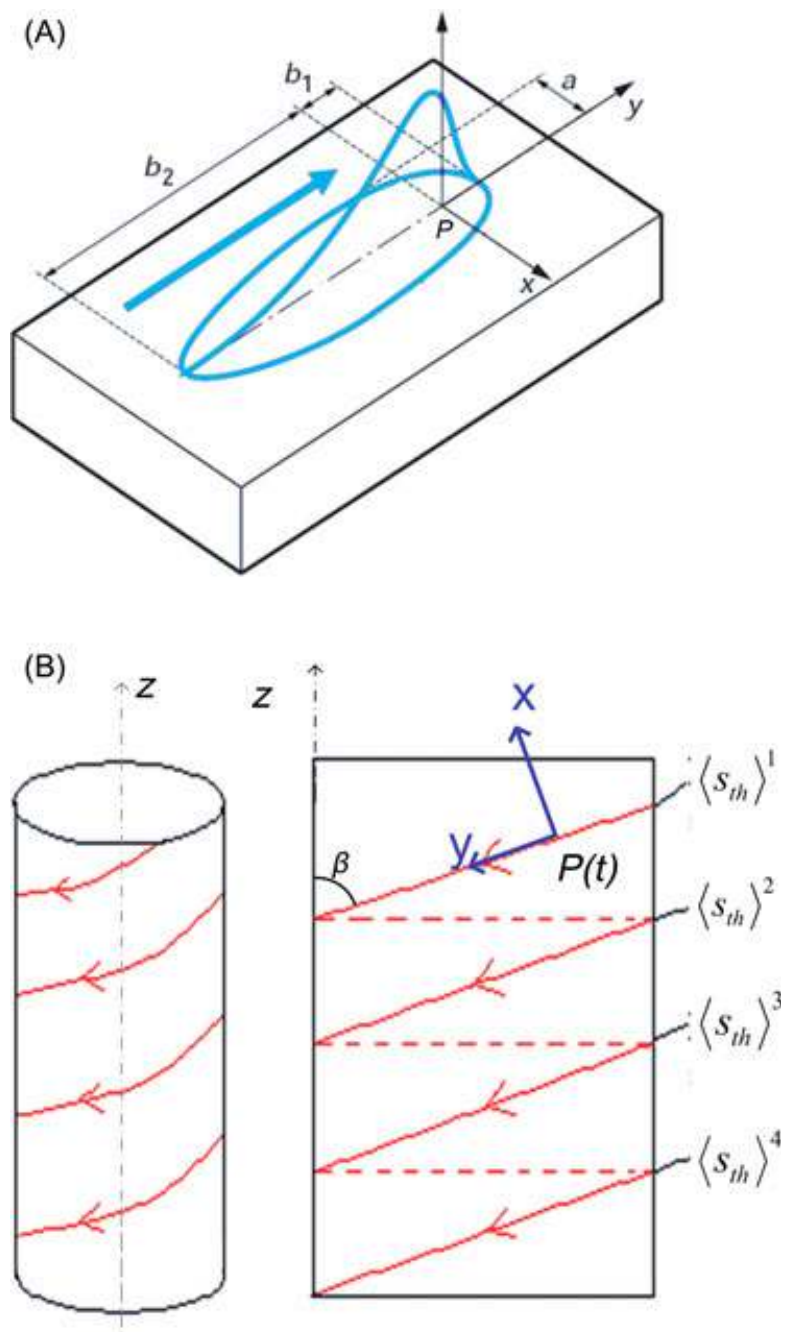

Figure 9: (A) Theoretical heat source distribution $\left\langle S_{t h}\right\rangle$; (B) heat source propagation and periodicity

This orientation is based on experimental temperatures field presented in Ref. [15]. An example with four heat sources bands is presented in Figure 9B.

The heat balance equation that is solved is identical to Equation (20), in which the radial heat losses by convection and radiation (characteristic time $\left.\tau_{t h}\right)$ were neglected. The transient boundary value problem was solved with the finite element software Comsol [14]. As shown in Figure 10A, a fine $2 \mathrm{D}$ mapped mesh made of $150 \times 100$ quadrilateral elements with second-order polynomial interpolation functions was used to run the simulation. The numerical results were then projected onto a Cartesian grid made of $77 \times 51$ points (see Figure 10B). Thereby, half the grid, i.e. a subset of $39 \times 51$ points (see Figure 10C), was extracted in order to select only the part of the tube observed by the infrared camera (view angle of $180^{\circ}$ ). Finally, as shown in Figure 10D, the last grid was projected

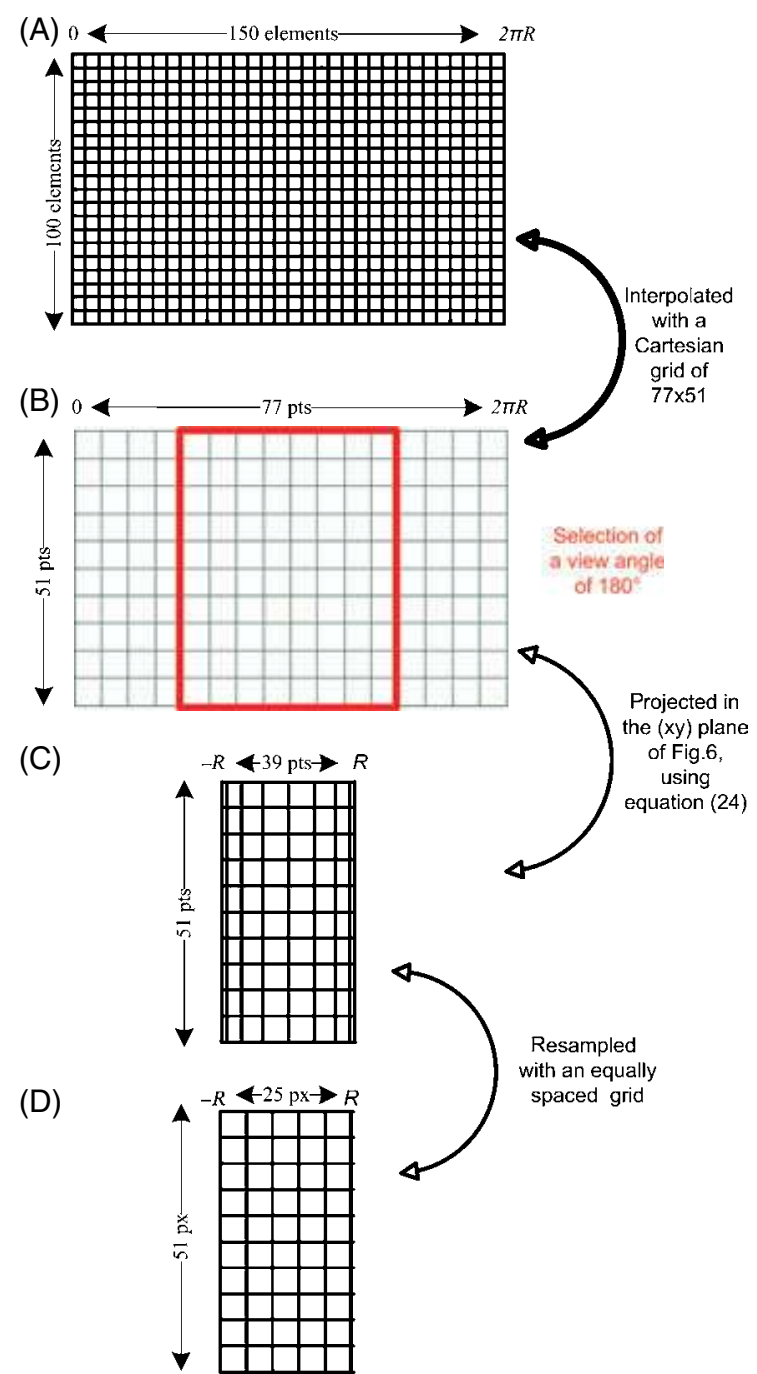

Figure 10: Scheme of the successive steps proposed to build a numerical thermal image. (A) 2D finite element mesh of the plate used to compute the thermal field. (B) Windowing operation applied to the transverse direction in order to select only a view angle of $180^{\circ}$. (C) Projection of the grid in the (xy) plane of Figure 6, using Equation (24). (D) Resampling of the grid, with an equally spaced mesh, in order to have a regularly thermal image, with $25 \times 51$ square pixels, as for real observations

in the (xy) plane of Figure 6, using Equation (24). It was also re-sampled with an equally spaced grid, to obtain virtual thermal images with $25 \times 51$ square pixels similar to those recorded by the infrared camera. These numerical thermal images will then be used, as input data, to check the validity of the software.

The NSTF results. As the filter parameters are very sensitive to the spatial sampling frequency and to the heat source characteristics, the NSTF's parameters have been selected close to the experimental data (Table 1). An example of results obtained at a given time $(1.5 \mathrm{~s})$, of the different fields described in 


\begin{tabular}{llllll}
\hline $\mathrm{V}_{S}\left(\mathrm{~m} \mathrm{~s}^{-1}\right)$ & $a(\mathrm{~m})$ & $b \mathrm{I}(\mathrm{m})$ & $b 2(\mathrm{~m})$ & $Q_{\max }\left(\mathrm{W} \mathrm{m}^{-3}\right)$ & Angle \\
\hline $0.0 \mathrm{II} 0$ & $0.00 \mathrm{II}$ & 0.0033 & 0.0830 & $2 \mathrm{e} 7$ & $59^{\circ}$ \\
$\Delta x=\Delta z(\mathrm{~mm})$ & $\Delta t(\mathrm{~s})$ & $T_{0}(\mathrm{~K})$ & $C\left(\mathrm{Jg}^{-1} \mathrm{~K}^{-1}\right)$ & $\rho\left(\mathrm{kg} \mathrm{m}^{-3}\right)$ & $k\left(\mathrm{~W} \mathrm{~m}^{-1} \mathrm{~K}^{-1}\right)$ \\
0.2477 & 0.1 & 297 & 490 & 6400 & $\mathrm{I} 5$ \\
\hline
\end{tabular}

Table I: Heat source distribution and other NSFT parameters

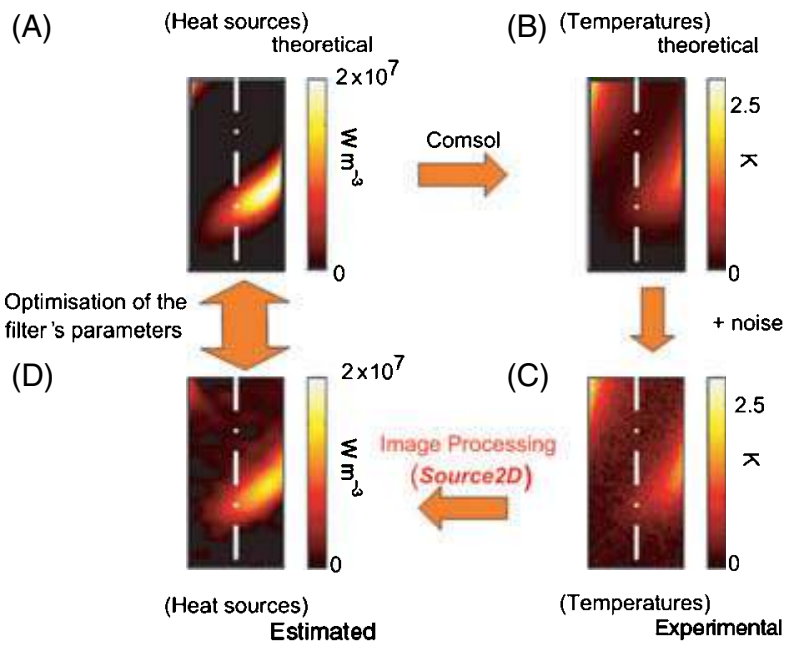

Figure I I: NSFT results at a current time (1.5 s). All the fields are projected on the tube surface as they are observed by the infrared camera: (A) theoretical (analytical) heat source field; (B) associated temperature variation field estimated by the finite element model; (C) noisy temperature field; (D) heat source field estimated by the image-processing software Source2D

Figure 7 is presented in Figure 11. A spatiotemporal representation of the NSTF results is also plotted in Figure 12. It presents the time evolution of an axial profile of the previous fields. Because of thermal conduction, the axial heat sources and temperature distributions are very different. These NSTF show and confirm the difficulty to interpret the observed temperature fields.

Using the filtering process, it is possible to estimate quantitatively heat sources: shape, position, intensity, number of bands, velocity, etc. In order to check the validity and to adjust the parameters of the processing, temporal evolutions or axial profiles (sections of the previous spatiotemporal images) are plotted in Figure 13A,C,E. Figure 13A,E show that heat sources are $20 \%$ underestimated in the centre of the band, which is due to the lowpass spatial filtering processing described before. Outside the band, the estimation is better. The Figure 13B,D,F shows that the estimation error is mainly induced during the Laplacian estimation, and not from the time-partial derivative which is rather well calculated. These figures also show the different filtering steps, for the Laplacian and for the time derivative.

Figure 14 underlines the influence of cutoff frequencies on the filtering process. For that purpose, five different increasing values for the filter's cutoff frequencies have been tested, ranging between filter 0 and filter 4 respectively. Depending on the spatial
(A) (Heat sources)

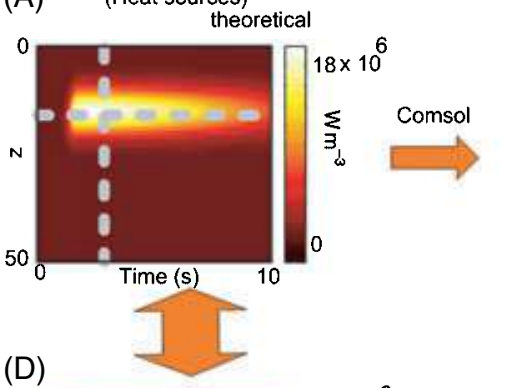

(D)

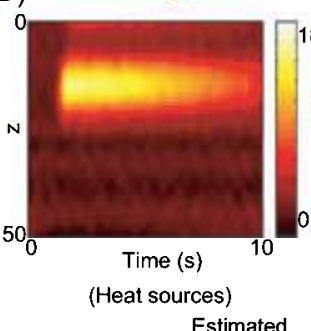

(B) (Temperatures)

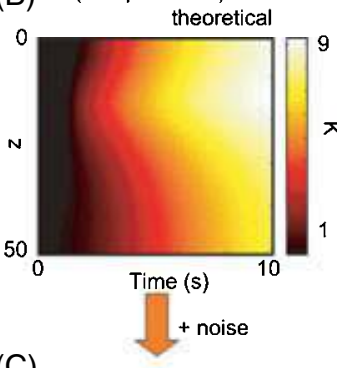

(C)

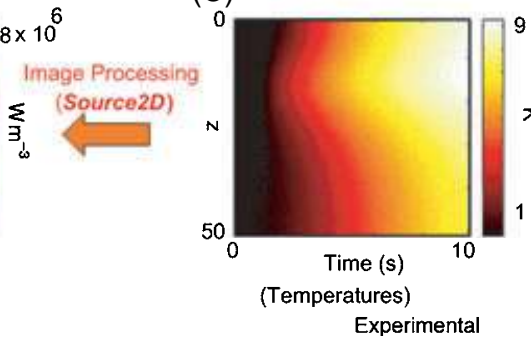

Figure 12: Spatiotemporal presentation of the NSFT results. Temporal evolution of an axial profile of: (A) theoretical heat sources; (B) associated temperature variation field estimated by the finite element model; (C) noisy temperature field; (D) heat source field estimated by the image-processing software Source2D 

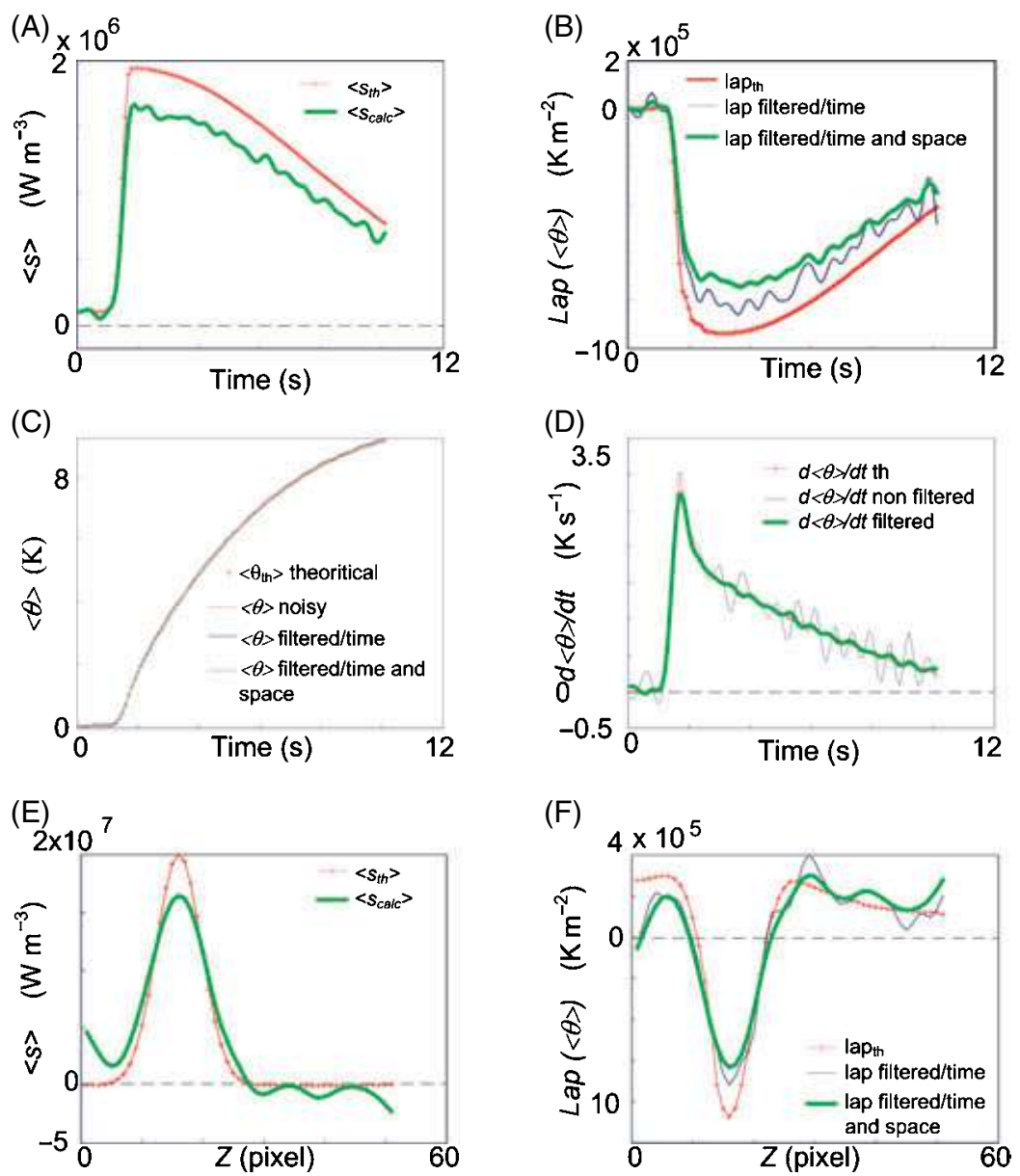

Figure 13: Temporal evolution, in pixel 17 (see horizontal white dot line in Figure 12A), of: (A) theoretical and estimated heat sources; (B) theoretical and estimated Laplacian operator with the two filtering steps; (C) theoretical and estimated temperature variations with the two filtering steps; (D) theoretical and estimated time derivative filtered and not filtered. Axial profiles, at time $t=30 \mathrm{~s}$ (see vertical white dot line in Figure 11A), of: (E) theoretical and estimated heat sources, (F) theoretical and estimated Laplacian operator with the two filtering steps

resolution (55 points in the axial direction) and on the heat source width, an optimal value has been fixed and corresponds to filter 2 . In this situation, the cutoff frequency equals 15\% of Nyquist frequency and tends to minimise ripples in the laplacian image (see filter 0 and 1 for example).
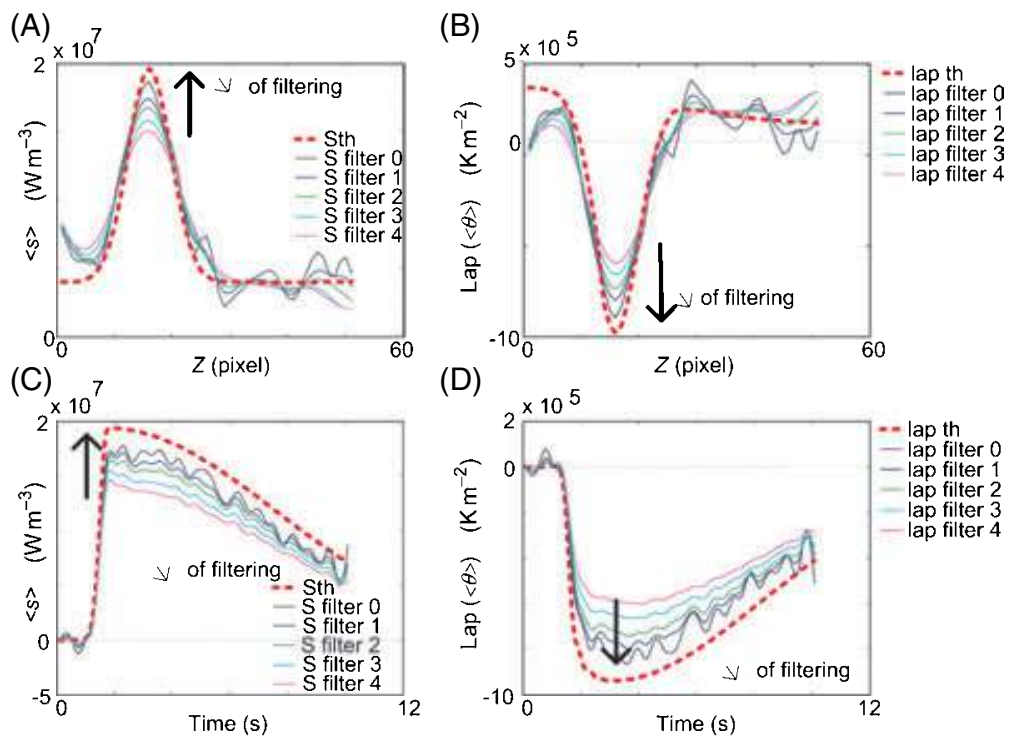

Figure 14: Influence of filtering for: (A) a heat source profile; (B) a Laplacian profile; (C) a temporal evolution of the heat source; (D) a temporal evolution of the Laplacian operator 


\section{Example of results}

The processing method can now applied to temperature fields which have been experimentally observed during the tensile test shown in Figure 4. Only one test at imposed nominal strain rate $\left(2.3 \times 10^{-4} \mathrm{~s}^{-1}\right)$ will be presented, on a 0.5 -mm-thick tube of NiTi as detailed in part 2 (Material, ...).

The acquisition parameters of this test are a spatial resolution $\Delta x=\Delta z=0.4 \mathrm{~mm}$ and an acquisition period $\Delta t=0.1 \mathrm{~s}$ which are close to the values used in the NSTF (cf. Table 1). Filter 2 was chosen with regard to the NSTF results presented in the previous section (Figure 14). The processing requires a knowledge of the heat capacity $C$ and of the thermal conductivity $k$, which are assumed to be homogeneous and constant. The experimental values of $C$ for NiTi alloy range from 450 to $600 \mathrm{~J} \mathrm{~kg}^{-1} \mathrm{~K}^{-1}$, whereas those for $k$ range from 8 to $28 \mathrm{~W} \mathrm{~m}^{-1} \mathrm{~K}^{-1}$ [13]. In this study, $C$ and $k$ were taken constant and equal to $490 \mathrm{~J} \mathrm{~kg}^{-1} \mathrm{~K}^{-1}$ and $15 \mathrm{~W} \mathrm{~m}^{-1} \mathrm{~K}^{-1}$, respectively.

The processing area is plotted in Figure 15A and covers a transverse width of 15 pixels taken between an orientation of $\pm 45^{\circ}$ to the optical axis. The observed temperature variation fields are plotted in the form of a spatiotemporal representation in Figure 15B. The temporal evolution of the nominal stress is superimposed, in black, in this image. First, we observe an almost homogenous temperature increase when the stress curve is almost linear. This increase in this zone suggests the occurrence of a stress-induced phase transformation: indeed, the opposite trend would have been expected with a purely elastic deformation mode (thermoelastic coupling). When the stress starts to decrease, the temperature variations are suddenly localised in a helical band which initiates in the upper grip and propagates at constant velocity to the bottom one. During the stress plateau, the temperature increases: $+14 \mathrm{~K}$ at the centre of the tube. When unloading, the temperature decreases progressively. At the end of the test the final temperature is $12 \mathrm{~K}$ less than the ambient initial temperature in the middle of the tube. This behaviour is explained by the endothermic character of the martensite-austenite transformation. The heat source results are presented in Figure 15C,D. Without the regularising effects of the heat diffusion, they clearly show a better contrast.

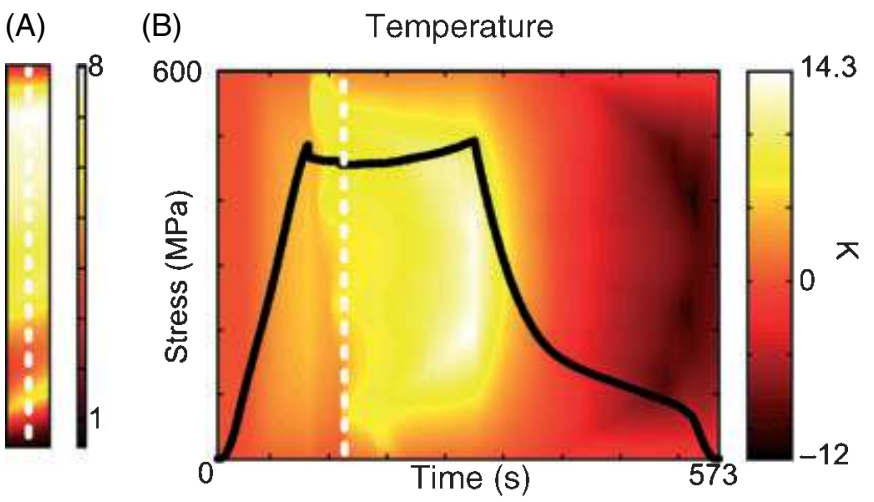

(B)

Temperature

(C) (D)

Heat source

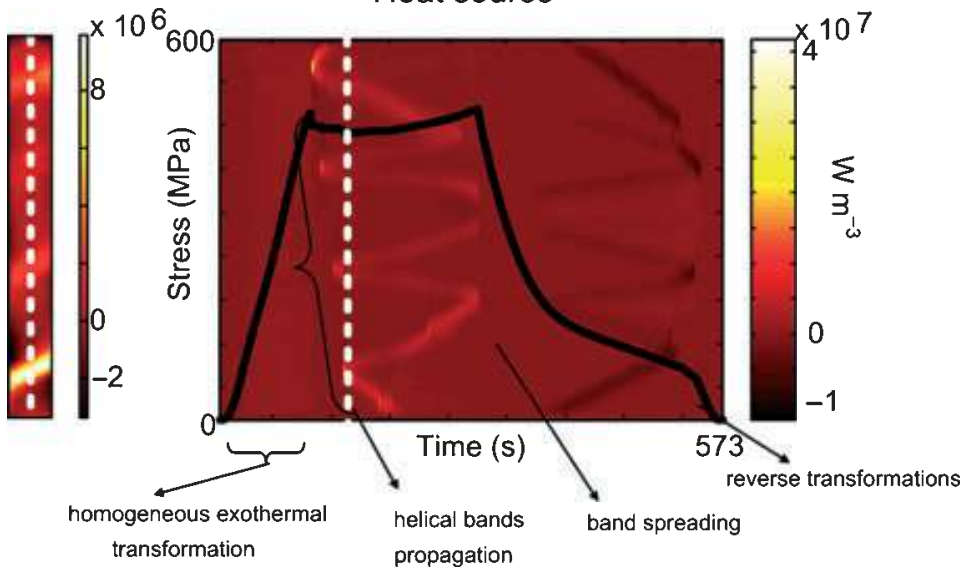

Figure 15: Experimental results: (A) temperature variation field observed at a fixed time given by the white dot line of (B); (B) spatiotemporal representation of an axial profile of observed temperature variation field; (C) observed heat sources field at a fixed time given by the white dot line of (d); (D) spatiotemporal representation of an axial profile of observed heat sources field. The temporal evolution of the nominal stress superimposed, in black to (B) and (D) 
In the first stage, the heat sources are positive and homogeneous (Figure 15D). The helical band appears at the yield point and, during the nominal stress decrease, propagates along the tube with a constant intensity and a constant velocity. Then, the oriented bands in this spatiotemporal representation, are associated to seven transformation fronts [16] which move, at different velocity, along the tube. At the end of the loading, the quasi-totality of the tube was crossed by a transformation front. During unloading, reverse endothermic transformations appear, first homogeneously, and then display localised transformation fronts in a helical band in the same place as the beginning of the test.

\section{Conclusions}

Temperature and strain fields have been observed during superelastic tensile tests on NiTi tubes. They showed strong localisations that take the shape of helical bands with characteristics that are dependent on global tensile strain and strain rate. In order to obtain quantitative energy information to allow a better recognition of the deformation mechanisms involved inside and outside the helical bands, an estimation of the local heat sources based on image processing of the temperature fields has been proposed. This processing method has been calibrated and validated on numerically simulated temperature fields. It has been applied to experimentally observed temperature fields during a typical superelastic tensile test of an NiTi tube. The technique has shown important differences between temperature variations and heat source fields, providing interesting information on the type behaviour of the material. During the loading stage, at first a homogeneous exothermic stage is observed, then the heat sources are organised in helical bands crossing the tube, then spreading across it. Finally, the reverse behaviour occurs which is endothermic and localised in the same type of bands, was observed during unloading.

The processing technique will now be applied to an existing database and the coupling with the kinematics fields measured during the tests will be addressed. This will allow a detailed analysis of the superelastic tests performed on NiTi SMA tubes. This analysis is crucial because these heterogeneous tensile tests are the bases to propose constitutive equations and to study the fatigue behaviour of these materials [17].

\section{ACKNOWLEDGEMENTS}

The support of Minitubes SA (Grenoble, France) during this study is gratefully acknowledged.

\section{REFERENCES}

1. Miyazaki, S., Imai, T., Otsuka, K. and Suzuki, Y. (1981) Lüders-like deformation observed in the transformation pseudoelasticity of a NiTi alloy. Scripta Metall. 15, 853-856.

2. Sun, Q. P. and Li, Z. Q. (2002) The initiation and growth of macroscopic martensite band in nano-grained NiTi microtube under tension. Int. J. Plasticity 18, 1481-1498.

3. Brinson, C. L., Schmidt, I. and Lammering, R. (2004) Stress-induced transformation behaviour of polycristalline NiTi shape memory alloy: micro and macromechanical investigations via in situ optical microscopy. J. Mech. Phys. Solids 52, 1549-1571.

4. Orgéas, L. and Favier, D. (1998) Stress-induced martensitic transformation of a NiTi alloy in isothermal shear, tension, and compression. Acta Mater. 46, 5579-5591.

5. Shaw, J. A. and Kyriakides, S. (1997) On the nucleation and propagation of phase transformation fronts in a NiTi alloy. Acta Mater. 45, 683-700.

6. Chache, M. (2004) Etude de l'écrouissage cyclique des matériaux métalliques et des phénomènes de rochet. PhD Dissertation, Université Joseph Fourier Grenoble I.

7. Vacher, P., Dumoulin, S., Morestin, F. and Mgil-Touchal, S. (1999) Bidimensional deformation measurement using digital images. Proc. Inst. Mech. Eng. (ImechE), 213C, 811-817.

8. Chrysochoos, A. and Louche, H. (2000) An Infrared image processing to analyse the calorific effects accompanying strain localisation. Int. J. of Eng. Sci. 38, 1759-1788.

9. Louche, H. (1999), Analyse par thermographie infrarouge des effets dissipatifs de la localisation dans les aciers, PhD Dissertation, Montpellier University.

10. Mc Cormick, P. G., Liu, Y. and Miyazaki, S. (1993) Intrinsic thermal-mechanical behaviour associated with the stress-induced martensitic transformation in NiTi. Mater. Sci. Eng. A167, 51-56.

11. Balandraud, X., Chrysochoos, A., Leclercq, S. and Peyroux, R. (2001) Influence of the thermomechanical coupling on the propagation of a phase change front. $C R$ Mécanique 329, Série IIb, 621-626.

12. Louche, H. and Chrysochoos, A. (2001) Thermal and dissipative effects accompanying Lüders bands propagation. Mater Sci. Eng. A. A307, 15-22.

13. Faulkner, M. G., Amalraj, J. J. and Bhattacharyya, A. (2000) Experimental determination of thermal and electrical properties of Ni-Ti shape memory wires. Smart Mater. Struct. 9, 632-639.

14. COMSOL 3.2 (FEMLAB). http://www.comsol.com/.

15. Favier, D., Louche, H., Schlosser, P, Orgéas, L., Vacher, P. and Debove, L. (2007) Homogeneous and heterogeneous deformation mechanisms in an austenitic polycrystalline Ti-50.8 at.\% $\mathrm{Ni}$ thin tube under tension. Investigation via temperature and strain fields measurements. Acta Mater., in press.

16. Feng, P. and Sun, Q. P. (2006) Experimental investigation on macroscopic domain formation and evolution in polycrystalline NiTi microtubing under mechanical force. J. Mech. Phys. Sol. 56, 1568-1603.

17. Gong, X., Pelton, A. R., Duerig, T. and Hall, A. (2004) Cyclic properties of superelastic nitinol tubing. Proc. Tech. Mater. Processes Med. Devices Conf., 25-27 August, St Paul, MN, USA, 26-31. 